\title{
Contributing factors for acquired muscle weakness in intensive care unit
}

\author{
Hayam Ibrahim Asfour *1,2 \\ ${ }^{1}$ Critical Care and Emergency Nursing, Department of Critical Care and Emergency Nursing, Faculty of Nursing, University of \\ Alexandria, Egypt \\ ${ }^{2}$ Faculty of Nursing, Umm Al-Qura University, KSA
}

Received: January 18, 2016

DOI: $10.5430 /$ jnep.v6n8p102
Accepted: March 17, $2016 \quad$ Online Published: April 1, 2016

URL: http://dx.doi.org/10.5430/jnep.v6n8p102

\begin{abstract}
Introduction: One of the complications of intensive care unit (ICU) admission is neuromuscular dysfunction because of critical illness, which may lead to disuse atrophy. Many of critically ill patients have severe muscle weakness, which has been termed ICU-acquired weakness (ICU-AW). ICU-AW has many consequences on patients' outcome. The aim of the study was to determine contributing factors for occurrence of acquired muscle weakness in intensive care units.

Setting: The study was conducted in four general ICUs in two selected Hospitals in Makkah in KSA.

Subjects: Hundred patients were included in the study.

Materials and Method: Tool one: factors contributing to ICU-AW assessment tool. Tool two: indicators of ICU-AW in critically ill patients.

Results: Forty-two patients were diagnosed ICU-AW. Differences between the ICU-AW group and the no ICU-AW group in, sex, diagnosis on admission and APACHE II were not statistically significant. Age in the ICU-AW group was higher than in no ICU-AW group [ $59 \pm 14$ vs. 53 \pm 11 years, $p=.0183]$ which was significant statistical difference.

Conclusion: The contributing factors for ICU-AW are immobility, patients' age, level of consciousness, parenteral nutrition, and glucocorticoid \& insulin therapy.
\end{abstract}

Key Words: Intensive care unit acquired weakness, Critical care, Nursing practices

\section{BACKGROUND}

Many medical conditions such as myocardial infarctions and surgical complications have been managed by prescribed immobility. There is a perception that critically ill patients' bed rest is needed to minimize oxygen consumption, improve oxygenation, maintain spine \& bone alignment, and minimize trauma. However, the adverse effects of bed rest on patients are well known. Therefore, early mobilization of critically ill patients can prevent complications. Contradict- ing for these evidences, there has been a shift in managing critically ill patients from awake and interactive to immobile and sedated patients for days, and sometimes for weeks. ${ }^{[1]}$

Studies have revealed that a significant proportion of survivors of critical illness have profound muscle weakness and consequent impairment in functional status and quality of life for years after hospital discharge. The development of muscle weakness has multi-factorial reasons, including premorbid conditions and ICU-acquired weakness (ICU-AW).

\footnotetext{
*Correspondence: Hayam Ibrahim Asfour; Email: hayamasfour2002@gmail.com; Address: Critical Care and Emergency Nursing, Department of Critical Care and Emergency Nursing, Faculty of Nursing, University of Alexandria, Egypt. 
ICU-AW is defined as a "syndrome of generalized limb weakness that develops while the patient is critically ill and for which there is no alternative explanation other than the critical illness itself". ICU-AW may develop because of critical illness, deep sedation, and prescribed immobility. ${ }^{[2-4]}$

ICU-acquired weakness is a common ICU complication. In a systematic review of 24 studies, $46 \%$ of critically ill patients had neuromuscular complications, which were associated with prolonged mechanical ventilation duration and increased ICU length of stay. In other prospective studies, the incidence of ICU-AW was $25 \%-58 \%$ for patients ventilated for at least 7 days. The incidence of ICU-AW has been reported to be as high as $50 \%$ to $100 \%$ among patients with sepsis. ${ }^{[1-5]}$

It has been thought that bed rest is necessary for recovery; prolonged immobility beyond 24 to 48 hours is associated with many detrimental physiological consequences. Bedbound patients sustain muscle atrophy from the loss of mechanical loading required to maintain muscle length. These unloaded muscles have fewer actin filaments leading to a lower force per cross sectional area, which manifests clinically as muscle weakness. In addition, unloaded muscles have decreased protein synthesis and accelerated protein degradation that resulted in muscle atrophy. Muscle atrophy may occur within hours of immobility in healthy subjects, as each week of bed rest leads to loss of muscle strength up to five percent. Immobility increases the production of pro-inflammatory cytokines and reactive oxygen species, resulting in further breakdown of muscle proteins, which increase muscle weakness. ${ }^{[2,3,6,7]}$

The interaction of immobility with critical illness can further accelerate muscle loss. Catabolism can be up to $2 \%$ per day in critically ill patients leading to decrease muscle fiber area by $4 \%$ per day with severe atrophy. ICU-AW has also been associated with severity of illness. In addition to, the systematic inflammatory response syndrome (SIRS) has a more profound impact on muscle loss than with immobilization alone. Sepsis decreased protein synthesis in the muscles leading to increase catabolism and decrease the muscle mass of the lower extremity. ${ }^{[8,9]}$

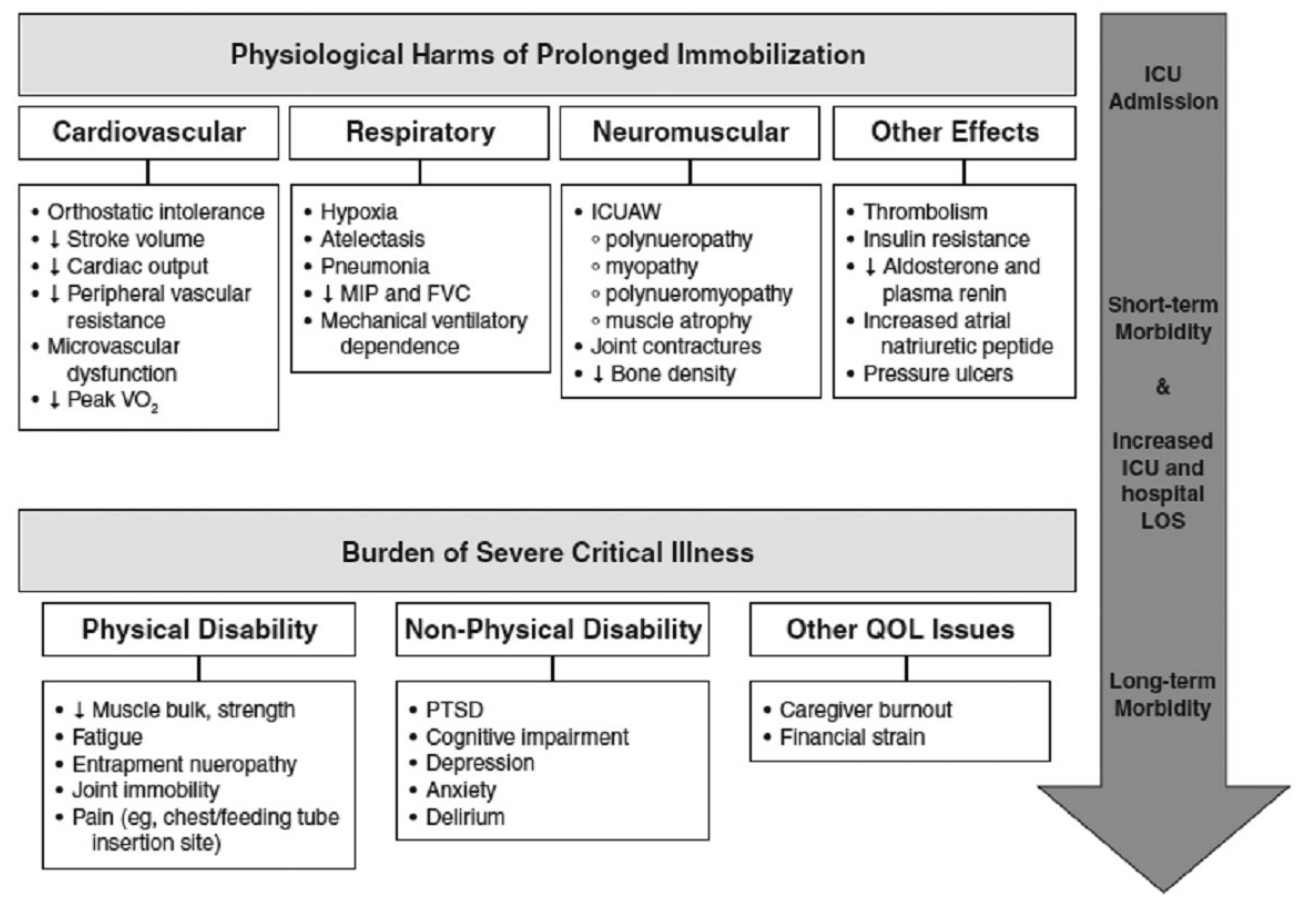

Figure 1. Physiological sequelae of immobilization and the burden of severe critical illness. FVC = forced vital capacity; ICU-AW = ICU-acquired weakness; MIP = maximum inspiratory pressure; PTSD = post-traumatic stress disorder; $\mathrm{VO}_{2}=$ oxygen delivery

Many factors make critically ill patients at risk for the development of ICU-AW, such as prolonged immobility, malnutrition, over-sedation, and systemic inflammation, but recent attention has focused on a number of controversial risk factors, which are neuromuscular blocking agents, corticosteroids,

Published by Sciedu Press

and hyperglycemia. Evidences are conflicting with respect to corticosteroids, neuromuscular blocking agents, and the development of ICU-AW. While a number of observational studies have not confirmed a significant association between corticosteroids and ICU-AW, analysis of a randomized con- 
trol trial (RCT) in respiratory failure, revealed occurrence of ICU-AW in patients with corticosteroid group. Analysis of two large randomized clinical trial had reported significant reductions in ICU-AW with tight glycemic control ${ }^{[3,10]}$ (see Figure 1).

For the diagnosis of ICU-AW, routine screening of all ICU patients with electro-physiological testing is not feasible because of challenges in testing and interpretation of the results in critically ill patients, as well as problems with cost and availability of equipment and expertise. The majority of studies advocate performing bedside physical examination of peripheral muscle groups using the Medical Research Council (MRC) scale sum score as a starting point for ICU-AW diagnosis. A composite MRC score from the examination of three muscle groups in each limb (range from no muscle contraction $=$ zero to normal strength $=5$ ) is commonly used, with $<80 \%$ of the maximum score (i.e., $<48$ out of 60 ) representing ICU-AW. MRC score has excellent inter-rater reliability in critically ill patients. ${ }^{[3-5]}$

Commonly, patients recovering from critical illness suffer from persistent impairments in health-related quality of life. Herridge et al. ${ }^{[12]}$ studied prospectively 109 ICU survivors and found persistent functional disability for five years af ter ICU discharge. These critically ill patients lost eighteen percent of their baseline body weight in the ICU and had severe muscle atrophy. Physical functional impairment was because of losing muscle bulk, muscle weakness, and fatigue as stated by these patients. After one year, over half of the patients could not return to work because they still experienced weakness, persistent fatigue and poor functional status. Therefore, critical illness may result in debilitating disability leading to ongoing requirements for medical care, financial strain, and caregiver burnout. ${ }^{[12]}$

Studies have evaluated early mobilization of critically ill patients over the last decade, as it is the important therapeutic option for prevention and treatment of ICU-AW..$^{[1,6,7,13-15]}$ ICU-AW preventive measures mainly depends on nursing preventive interventions, that include main items describing nursing measures to promote patients mobilization as positioning, active and passive range of motion exercises, changing position, chair sitting and walking. Critically ill patients early mobilization will increase muscle strength, and prevent muscle atrophy. Moreover, mobilization and exercise may promote health because they can prevent insulin resistance and microvascular dysfunction and decrease oxidative stress \& inflammation, which may lead to a reduction in ICU-AW ${ }^{[1,6,7,13-15]}$.

In recent years, attention for patients' safety has increased, however the focus of this attention is on the epidemiology of errors and adverse events, not on preventive practices to reduce such events. Recently, the safety and feasibility of early mobilization of critically ill patients are documented in a number of studies. In addition, early mobilization and rehabilitation are associated with important decrease in mechanical ventilation duration, and improved physical function at hospital discharge. Despite the results of these studies, early mobilization is performed for only about a quarter of all critically ill patients. ${ }^{[13-18]}$

Critical care nurses have important roles; firstly as patients' advocate to maintain patients' safety and prevent harm, and to promote health. Therefore, identifying patients at risk for ICU-AW is important which can be done by performing assessment and physical examination especially musculoskeletal examination and promoting patients' health through positioning, turning, performing range of motion exercise (passive and active). Hence, the current study aimed to identify the factors contributing to ICU-AW in critically ill patients.

\subsection{Aim of the study}

To determine contributing factors for occurrence of acquired muscle weakness in intensive care units.

\subsection{Study question}

What are the contributing factors for occurrence of acquired muscle weakness in intensive care units?

\section{MATERials AND METHOD}

\subsection{Research design}

The study was conducted following a descriptive design.

\subsection{Setting}

Four general ICUs in two selected Hospitals in Makkah in KSA were the settings in which the current study conducted.

\subsection{Subjects}

Hundred patients were consecutively screened for eligibility after 48 hours in the ICU. Inclusion criteria; patients above 18 years, mechanically ventilated for more than 3 days, did not have neuromuscular disease, able to follow simple commands, and with an APACHE II admission score $\geq 13$. Patients with preexisting neuromuscular disease (e.g. myasthenia Gravis, Guillain-Barre), spinal cord injury, stroke, diseases with systemic vascular involvement such as lupus erythematosus, obesity (BMI $>35 \mathrm{~kg} / \mathrm{m}^{2}$ ), and with neuromuscular blocking agents, inability to provide informed consent were excluded from the study.

\subsection{Tools}

The researchers developed two tools after reviewing the relevant literatures. $[1,3,7,8,10,11]$ 
Tool one: Factors contributing to Intensive Care Unit Acquired Weakness (ICU-AW) assessment tool. The researcher developed this tool after reviewing the related literatures ${ }^{[1,3,7,8,10,11]}$ to assess contributing factors for the occurrence of acquired muscle weakness in intensive care units. It consists of two parts:

Part (I): ICU-AW preventive measures observational checklist". A checklist was used to observe ICU-AW preventive measures. It contains five main items describing nursing preventive measures as positioning, active and passive range of motion exercises, changing position, chair sitting and walking. Nursing preventive measures were measured using a scale of done, and not done with scores one and zero respectively. The scores for the individual items were then summed up to obtain the total score.

Part (II): Drug-related factors. It includes prescribed medications during the ICU stay such as glucocorticoid therapy and insulin therapy. The response format is measured using scale of yes (present), no (not present).

In addition, patients' characteristics which are sex, age and name of the ICU and clinical data in relation to ICU-AW such as the admission medical diagnosis, co-morbidities, LOS, APACHE II score, methods of nutritional support, duration on mechanical ventilation, serum transferrin, serum pre-albumin, and total protein.

Tool two: ICU-AW indicators in critically ill patients. This tool was used to monitor critically ill patients' indicators of ICU-AW in critically ill patients. It consists of two parts:

Part (I): "The Medical Research Council (MRC) scale". This scale is used for assessment of muscle strength clinically which is used by many studies. ${ }^{[3-5]}$ These studies clearly stated an MRC score threshold to define significant weakness. Therefore, MRC score was used in the current study to diagnose ICU-AW. The diagnosis of ICU-AW is made with grading muscle strength in the upper and lower extremities. The assessment of muscle strength include; wrist extension, elbow flexion, and bilateral shoulder abduction, for the upper limbs. For the lower limps include hip flexion, knee extension and foot dorsiflexion.

Scoring; muscle strength in each group was scored according to the six-point MRC system, which are; no visible contraction which was given zero, visible contraction without movements of the limbs equal one, movements of the limbs but not against the gravity equal two, movement against gravity over (almost) the full range was given three, active movement against gravity and resistance was given four and five was scored for presence of normal muscle power.

Published by Sciedu Press
The scale of MRC is ranging from zero to 60 . The higher scores indicating greater muscle strength. Presence of ICUAW is considered when the total score is less than 48. The cut off limit of 48 for the MRC indicates clinically significant weakness and it has been used previously for the clinical identification of ICU-AW. ${ }^{[3-5]}$

Part (II): "Intensive Care Unit Acquired Weakness (ICU-AW) related outcomes". The researcher developed this part based on reviewing the related literatures. ${ }^{[2,3,6,10,11,15]}$ It was used to record the clinical findings/outcomes occurred to patients because of ICU-AW. These outcomes/findings include; weaning difficulties from mechanical ventilation discharge from ICU and/or death. Findings/outcomes are measured using scale of Yes (present), No (not present).

\subsection{Tools validity and reliability}

The study tools were developed after reviewing the related literatures except tool 2 part I which was developed by the Medical Research Council. ${ }^{[3-5]}$ The tools were submitted to a Jury of 7 experts in critical care medicine, and critical care nursing to test the face and content validity of the tools, the necessary modifications were carried out according to the academic nursing experts' judgment on clarity of sentences and the appropriateness of the content. Tools reliability was tested using internal consistency methods (Alpha Cronbach test). The reliability coefficients were $(r=0.788)$ which indicates an accepted reliability of the tools.

\subsection{Pilot study}

A pilot study was conducted on $10(10 \%)$ patients. It was selected from the previously mentioned study setting according to inclusion criteria to assess the current study tools for its clarity, validity, applicability and the time required to fill the tool. Necessary modifications of the tool were done according to pilot results to reach the finalized form. The subjects who included in the pilot study were excluded from the total study sample.

\subsection{Procedure}

The study included newly admitted patients who were selected according to the previously mentioned inclusion criteria, then patients' characteristics such as age, sex and the name of ICU, in addition to patients' clinical data such as admission medical diagnosis, co-morbidities, hemodynamically stability, APACHE II score, duration on mechanical ventilation, serum transferrin, serum pre-albumin, and total protein level; were recorded upon the admission using tool one. The researcher trained two-research assistances to aid in data collection. (in observing intensive care unit acquired weakness preventive measures only). Inter-observer consistency exceeding $90 \%$ was confirmed before the study. 
Firstly, hemodynamically stability were assessed for patients (MAP $\geq 60 \mathrm{mmHg}, \mathrm{SPO}_{2} \geq 90$, HR 60-120 b/m). On ICU admission, a dietitian assessed patients' nutritional status and prescribed the needed nutrition while nurses administered the nutrition as ordered. Then, intensive care unit acquired weakness preventive measures provided to patients were observed and recorded by the researcher using tool 1 part $I$ in three shifts using tool 1 part I, mainly; positioning, changing position, active and passive range of motion exercises (ROM), chair sitting and walking. Each patient was observed for 24 hours throughout the three shifts: morning, evening and night shift for ten days. Preventive measures were recorded using a scale of done, and not done with scores one and zero respectively.

Patients were screened once daily for ICU-AW defined as the presence of symmetric and flaccid weakness associated with decreased or absent deep tendon reflexes using tool two part I (MRC) scale. Three muscle groups in all four limbs were assessed with MRC scale with values ranging from 60 (normal muscle strength) to zero (quadriplegia). Assessment of muscle strength was done from admission till patients discharge, transfer from ICU or death.

The following functions were assessed: wrist flexion, elbow flexion, shoulder abduction, ankle dorsiflexion, knee extension, hip flexion. Patients with an MRC score $<48 / 60$ were diagnosed with ICU-AW.

\subsection{Administrative design and ethical considerations}

The study conducted over a period of 12 months starting from April 2014 to April 2015. An official letter clarifying the purpose and setting of the study was obtained from the ethical committee and the head of critical care and emergency nursing department, Faculty of Nursing - Alexandria University - Egypt, and from the hospital authority to conduct the study. written informed consent was obtained from each patient. It included the aim of the study, potential benefits, risks and discomforts from participation. The confidentiality, anonymity \& privacy of responses were assured. The right to refuse to participate in the study was emphasized to subjects.

\subsection{Statistical analysis}

Data were entered and analyzed using SPSS version 17. The normality of data distribution was examined using Kolmogorov - Smirnov test. Descriptive statistics were used to summarize the categorical variables. Moreover, the significance of results of qualitative variables were tested using Chi-Square test and Fisher's exact test. Statistical significance was assessed using a $p$ value of $<.05$ and highly significant results was considered at $p<.01$.

\section{RESUlts}

The study included one hundred patients, who were selected according to the previously mentioned inclusion criteria. All patients had medical diagnoses. The ratio of nurse-to-patient ratio was 1:2 in all ICUs. One respiratory therapist was responsible in each unit for 24 hours. A dietitian assessed patients' nutritional status and prescribed the needed nutrition from the first day of ICU admission but nurses did the administration of nutritional support. Protocols, nursing staff and respiratory therapists work were similar across the study time.

Forty-two patients were diagnosed ICU-AW of the hundred patients. Differences in sex, diagnosis on admission and APACHE II score between the ICU-AW group and the no ICU-AW group were not statistically significant. Age of patients in the ICU-AW group [59 \pm 14$]$ was higher than in the no ICU-AW group [53 \pm 11 years, $p=.0183]$ which was significant statistical difference.

Table 1. Characteristics of critically ill patients on admission

\begin{tabular}{|c|c|c|c|}
\hline & $\begin{array}{l}\text { ICU-AW } \\
(n=42)\end{array}$ & $\begin{array}{l}\text { No ICU-AW } \\
(n=58)\end{array}$ & $p$ value \\
\hline \multicolumn{4}{|l|}{ Patient characteristics } \\
\hline Age $(\mathrm{y})$, mean $\pm S D$ & $59 \pm 14$ & $53 \pm 11$ & $.0183^{*}$ \\
\hline Sex; Male, n (\%) & 28 (66.67) & $42(72.41)$ & .6590 \\
\hline Sex; Female, n (\%) & $14(33.33)$ & $16(27.59)$ & \\
\hline \multicolumn{4}{|l|}{ ICU admission characteristics } \\
\hline \multicolumn{4}{|l|}{ Admission diagnosis } \\
\hline Cardiovascular, n (\%) & $12(28.57)$ & $26(44.82)$ & .6158 \\
\hline Respiratory, n (\%) & 11 (26.19) & 19 (32.75) & \\
\hline Neurological, n (\%) & $6(14.38)$ & 0 & \\
\hline Endocrine/metabolic, n (\%) & $4(9.52)$ & $4(6.89)$ & \\
\hline Gastrointestinal, n (\%) & 0 & $3(5.17)$ & \\
\hline Renal, n (\%) & $7(16.67)$ & $3(5.17)$ & \\
\hline Other, n (\%) & $2(4.67)$ & $3(5.17)$ & \\
\hline \multirow{2}{*}{\multicolumn{4}{|c|}{$\begin{array}{l}\text { Lab investigations Serum Transferrin, Serum Pre-Albumin/Total } \\
\text { Protein. }\end{array}$}} \\
\hline & & & \\
\hline Normal, n (\%) & $20(41.67)$ & $38(65.51)$ & \\
\hline Abnormal, n (\%) & $22(52.38)$ & $20(34.49)$ & \\
\hline \multicolumn{4}{|l|}{ Nutritional support } \\
\hline Enteral, n (\%) & 12 (28.57) & $58(100)$ & $0 *$ \\
\hline Parenteral, n (\%) & $30(71.42)$ & 0 & \\
\hline Enteral \& parenteral, $n(\%)$ & 0 & 0 & \\
\hline \multicolumn{4}{|l|}{ Medications } \\
\hline Glucocorticoid Therapy, n (\%) & $8(19.04)$ & 0 & $.0384 *$ \\
\hline Insulin Therapy, n (\%) & 20 (41.67) & $12(20.64)$ & \\
\hline APACHE II, mean $\pm S D$ & $16 \pm 5$ & $17 \pm 4$ & .2696 \\
\hline
\end{tabular}

Note. APACHE II, Acute Physiology and Chronic Health Evaluation II; ARDS, ICU-AW, ICUacquired weakness; *significant at $p<.05$

Number of patients who had abnormal serum transferrin, serum pre-albumin and total protein values was higher in ICU-AW group [22 (52.38\%)] than in no ICU-AW group [20 (34.49\%)]. As for methods of nutritional support, all patients in the no ICU-AW group were administered enteral 
nutrition compared with [12 $(28.57 \%)$ enteral nutrition and $30(71.42 \%)$ parenteral nutrition] in ICU-AW group, which was statistically significant. Regarding medications, it was noted that only $12(20.64 \%)$ patients in the no ICU-AW group were administered insulin therapy compared with [20 $(41.67 \%)$ insulin therapy and $8(19.04 \%)$ glucocorticoid therapy] in ICU-AW group which was statistically significant $(p=.0384)$. Baseline and ICU admission characteristics for critically ill patients are presented in Table 1.

ICU-AW preventive measures included positioning, turning, passive ROM and active ROM. These measures should be performed/assisted by the bedside nurses. Table 2 reveals the adequacy of ICU-AW preventive measures; It can be seen that the adequacy of positioning and turning in ICU-AW group $(33.33 \%, 22.22 \%)$ was lower than the no ICU-AW group $(66.67 \%, 55.55 \%)$ respectively. Patients of ICU-AW group were positioned on Semi-Fowler position all the day except the time of bathing; they were in supine position. While patients of no ICU-AW group were in different positions during the day. No patients ICU-AW group had passive ROE, active ROE, chair position or ambulation, while the no ICU-AW group had assisted in performing these activities therefore, the percent of adequacy for these activities were varies according to patients' participation $(50 \%, 77.5 \%, 20 \%$, $4 \%$ ) respectively.

Table 2. Adequacy of Intensive Care Unit Acquired Weakness (ICU-AW) Preventive Measures

\begin{tabular}{lll}
\hline \multirow{2}{*}{ Preventive Measures } & \multicolumn{2}{l}{ Level of practices adequacy (\%) } \\
\cline { 2 - 3 } & ICU-AW (n = 42) & No ICU-AW (n = 58) \\
\hline Positioning & 33.33 & 66.67 \\
Turning & 22.22 & 55.55 \\
Passive ROM & Zero & 50 \\
Active ROM & Zero & 77.5 \\
Chair position & Zero & 20 \\
Ambulation & Zero & 4 \\
\hline
\end{tabular}

Note. ICU-AW, ICU-acquired weakness, Passive ROM; passive range of motion exercise, Active ROM; active range of motion exercise.

Table 3, shows post ICU admission outcomes for critically ill patients. Regarding muscle strength; MRC score on patients' admission after stabilization, was lower in patients with ICU-AW group [50 \pm 8 ] than in the no ICU-AW group $[55 \pm 6]$ which was statistically significantly $[p=.0006]$. Patients with ICU-AW group had significantly lower muscle strength than no ICU-AW group at ICU discharge [35 \pm 6 vs. $47 \pm 7, p=.0001]$. Patients with ICU-AW had long duration of mechanical ventilation compared with others $[(20 \pm 10)$ vs. (14 \pm 7 ) days, $p=.0006]$. In addition, number of patients with ICU-AW group had weaning difficulties was higher than others, which was statistically significant [25/42 (59.52\%)

Published by Sciedu Press vs. $5 / 58(8.62 \%), p<.0001]$.

Table 3. Post ICU admission outcomes for critically ill patients

\begin{tabular}{llll}
\hline & $\begin{array}{l}\text { ICU-AW } \\
(\mathbf{n = 4 2 )}\end{array}$ & $\begin{array}{l}\text { No ICU-AW } \\
(\mathbf{n}=\mathbf{5 8})\end{array}$ & p value \\
\hline $\begin{array}{l}\text { MRC score, mean } \pm S D \\
\text { On admission }\end{array}$ & $50 \pm 8$ & $55 \pm 6$ & $.0006^{*}$ \\
$\begin{array}{l}\text { On discharge } \\
\text { Mechanical ventilation support }\end{array}$ & $55 \pm 6$ & & $<.0001^{*}$ \\
$\begin{array}{l}\text { Duration on Mechanical } \\
\text { ventilation, mean } \pm S D\end{array}$ & $20 \pm 10$ & $14 \pm 7$ & $.0006^{* *}$ \\
$\begin{array}{l}\text { Weaning difficulties, } \\
\text { n (\%) }\end{array}$ & $25(59.52)$ & $5(8.62)$ & $<.0001^{* *}$ \\
$\begin{array}{l}\text { LOS, mean } \pm S D \\
\text { Discharge, n (\%) }\end{array}$ & $24 \pm 8$ & $10 \pm 3$ & $<.0001^{* *}$ \\
ICU Mortality, n (\%) & $27(64.28)$ & $53(91.38)$ & .2829 \\
\end{tabular}

Note. ICU-AW, ICU-acquired weakness; MRC score, Medical Research Council score; LOS, Length of stay; ICU Mortality, intensive care unit mortality; *significant at $p<.05$; **highly significant at $p<.01$

As for length of ICU stay (LOS); patients who developed ICU-AW group had a longer ICU stay (24 \pm 8 ) days compared to patients who did not develop ICU-AW group (10 \pm 3$)$ days, which was statistically significant $[p<.0001]$. ICU mortality rate was higher in patients with ICU-AW group $[15 / 42$ $(35.71 \%)]$ than in the no ICU-AW group [3/85 (5.17\%)] which was statistically significant $[p=.0016]$. In addition, discharge rate from ICU was lower in patients with ICU-AW group [27/42 (64.28\%) vs. 53/58 (91.38\%); $p=.2829]$ which was not statistically significant.

\section{Discussion}

Prolonged bed rest plays a significant role in occurrence of ICU-AW and physical dysfunction. As critical care, nurses must deliver comprehensive, compassionate, complex, technological care. Nurses must provide care processes and practices without causing harm to patients. Several complications including ventilator-associated pneumonia, cognitive and functional limitations can be reduced by successful mobilization of critically ill patients early as ICU-AW, which may be burden up to five years after discharge. Therefore, the aim of this study was to determine contributing factors for occurrence of acquired muscle weakness in intensive care units.

From the current study findings, it can be noted that forty two percent of patient developed ICU-AW. Sharshar et al. ${ }^{[13]}$ agreed with these findings and reported the occurrence of ICU-AW in $46 \%$ patients with prolonged mechanical ventilation or syndrome of multiple organ dysfunction. Other researchers estimated that ICU-AW affects around sixty percent of ICU patients. ${ }^{[3,6,10]}$ Nanas et al. ${ }^{[18]}$ added that ICU-AW is a neuromuscular complication, which occurs 
frequently with critical illness, with an incidence of thirty to ninety percent based on the time and method of diagnosis. Patients in the current study admitted with different diagnosis and there is not significant difference regarding patients' diagnosis on admission between ICU-AW group and Non ICU-AW group. While, many researches had linked the occurrence of ICU-AW with patients with respiratory failure, sepsis, acute renal failure and hematological failure. In the current study, the age of patients' with ICU-AW was larger than others. This may be related to the physiological changes occurring with aging.

Malnutrition is considered an important contributing factor to the occurrence of ICU-AW. Providing nutritional support is an essential intervention in ICU to correct the nutritional deficit. The present study revealed that parenteral nutrition were given in ICU-AW group higher than in the no ICU-AW group who were administered enteral nutrition more. In this line, Puthacheary et al. ${ }^{[19]}$ stated that occurrence of muscle wasting was linked with increased delivery of protein in the ICU during the first week. In addition, Hermans et al. ${ }^{[20]}$ stated that during the first week in the ICU parenteral nutrition should be avoided as it enhanced recovery and decreased the incidence of weakness, comparing with early supplementation of enteral feeding. ${ }^{[20]}$ Needham et al. ${ }^{[21]}$ added that during the first week in the ICU, no detrimental effects was noted with enteral feeding on physical function up to 1 year following ARDS. ${ }^{[21]}$

Malnutrition is an important cause for loss of muscle mass in critically ill patients. As critically, ill patients may receive calories less than required for their needs. Malnutrition in conjunction with the increased metabolism because of the pathophysiological mechanism leads to a significant protein loss, which in turn decrease the lean body mass causing muscle atrophy. The link between parental nutrition and ICU-AW may be attributed to the using of lipids infusions that may have detrimental effects on peripheral nerves.

It was noted that ICU-AW group was administered glucocorticoid and insulin therapy more than others were. Corticosteroids may have a dangerous role on muscle mass in humans in general because of their catabolic effect. Therefore, many studies examined the role of corticosteroids on ICU-AW occurrence. ${ }^{[2-4,11,19-21]}$ Even non-hospitalized patients who receive corticosteroids are also liable to develop muscle atrophy. In addition, patients received insulin therapy to manage hyperglycemia that may worsen the microcirculation leading to generalized muscle weakness. Therefore, Hermans et al. ${ }^{[20]}$ and others ${ }^{[3,19,21]}$ stated that late initiation of parenteral nutrition and strict glycemic control may prevent development of ICU-AW.
The findings of the current study revealed that the MRC score of ICU-AW group was significantly low compared with the no ICU-AW group on admission and at ICU discharge. It also can be noted that MRC score for both groups decreased while their stay on the ICU. The noted decline in MRC score may be attributed to many factors such as patients' pathological state and immobility. In this line, Morris ${ }^{[22]}$ stated that occurrence of muscle wasting is highest during the first weeks of ICU stay (especially 2 to 3 weeks) and immobilization is considered the leading cause for it.

Mobility is a basic nursing care, which is essential in maintaining patients' safety and prevention of immobility complications. As mobility can improve gas exchange, decrease incidence of ventilator-associated pneumonia, decrease the duration of mechanical ventilation, and decrease the length of hospital stay. The current study findings revealed that the adequacy of practices related to promotion of mobility are low in general and specifically lower in ICU-AW group than others. Many studies agreed with the findings of the current study. Winkelman ${ }^{[23]}$ et al. in 2005, conducted an observational study over an 8-hour period in ICU, and found that only $2.7 \%$ of patients had position changes every 2 hours, and more than half of patients were supine for 4 to 8 hours.

In the opposite side, Sage Products Inc. ${ }^{[24]}$ conducted a survey on 173 nurses which aimed to determine nurses compliance to turning and repositioning protocols in their hospitals. The results of the survey showed that more than three quarters of nurses have a turning and positioning protocol in their facilities, while the vast majority of nurses reported that turning/repositioning patients is done every 2 hours.

Ahren ${ }^{[25]}$ et al. also evaluated the pulmonary functions of patients treated with continuous lateral rotation therapy (CLRT), compared with patients treated with standard turning techniques. The researchers reported that the pulmonary functions of patients treated with CLRT improved significantly than others. In addition, the occurrence of ventilatorassociated pneumonia and atelectasis were decreased with CLRT. Numerous studies support using CLRT for mechanically ventilated patients within the first two days of ICU admission. ${ }^{[26-30]}$ It is recommended that, rotation should be performed with a 40 degree turn on each side for 18 to 24 hours a day. Therapeutic positioning strategies increase the overall patients' compliance and improve short- and longterm patient outcomes. ${ }^{[24-27]}$

Positioning, repositioning, turning and ROE can be challenging in critically ill patients, and is considered time-consuming from the nursing point of view. The results of the current study may be due to many factors such as nurses; perception, and severity of patients' conditions. As nurses have 
the perception that, bed rest is necessary for critically ill patient to improve oxygenation and ventilation, minimize oxygen consumption, and maintain spine and bone alignment. Nurses may also consider many therapeutic-related factors as the safety of tubes and lines and patients' hemodynamic instability.

Patients' safety during mobilization is an important factor especially in patients with hemodynamic instability. A prospective cohort study was conducted on 103 patients with mechanical ventilation, aimed to determine whether it is safe to mobilize critically ill patients or not. In this study, patients had received 1,449 activities (turning, positioning, ambulating and exercise) and the patients' responses and adverse events (hypertension/hypotension, desaturation, falling, removal of lines/tubes, and extubation) were observed and documented. The researchers concluded that although occurrence of adverse events in less than one percent of patients, mobilization of critically ill patients with hemodynamic instability early was safe and improved patients outcome as $69 \%$ of patients had the ability to ambulate on ICU discharge (at least $30 \mathrm{~m}){ }^{[14]}$

Bed rest indorses immobilization of muscle fibers leading to decrease shortened length muscle fibers and connective tissues, resulting in loosing connective tissue in muscles and around the joint. This in turn makes the connective tissue denser and impair the function of limbs. If passive exercise was not performed frequently, and positioning/turning was not done properly for patients on bed rest, a firm contracture would be produced after 2 to 3 weeks of immobility. ${ }^{[1-3,11,28]}$ Therefore, studies recommend that, passive range of motion to be performed twice daily, to prevent contracture. ${ }^{[2,28]}$

\section{Mobility Assessment for Readiness}

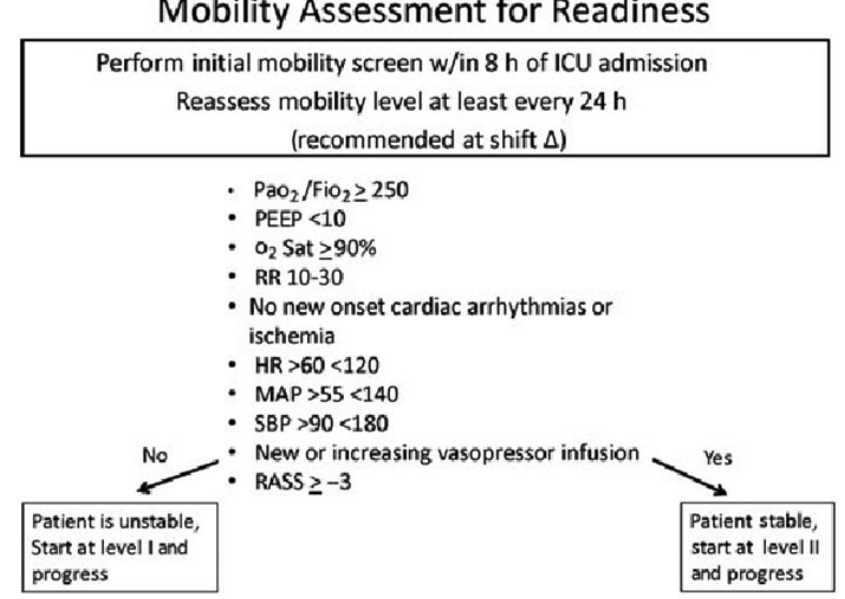

Figure 2. Assessment of patients' readiness for mobility

In addition, Morris et al. ${ }^{[31]}$ and Harvey ${ }^{[32]}$ recommended Published by Sciedu Press
ROM for all extremities to be performed 3 times a day, with a minimum of five repetitions/exercise. Functional outcome in ICU survivors at hospital discharge can be improved by daily exercise at of all limbs at bed level alone. However, nurses and hospital administrators should view mobilizing critically ill patients along a progressive continuum depending on many elements, which are; assessment of mobility readiness, patients' diagnosis, strategies to prevent complications, and patients' ability to tolerate the activity/movement. One of the critical care nurses role is to combat the occurrence of physical deconditioning caused by bed rest. Patients' readiness for mobility should be assessed daily ${ }^{[8]}$ (see Figure 2).

Many factors is affecting moving the immobilized patient and should be considered. Patients' related factors such as diagnosis, sedation, weight, and patients' complains as pain and discomfort. Nursing and administrative related factors such as nurse patient ratio, personnel and equipment resources mobilization policy and valuing, and priority of mobilization. Evidences suggested that a culture change is needed within the hospitals to encourage for early mobilization and incorporate it in the care of critically ill patients. ${ }^{[31-33]}$

Implementing mobility activities and exercises from patient admission and advancing each day throughout the hospital stay is called early progressive mobility. The mobility activities begin even if the patient is on vasopressors or mechanical ventilation. The activities start with turning, passive range of motion (ROM), and elevation of head of bed to 30 degree or greater, unless contraindicated, progressed to active ROM, advancing the bed to the chair position, dangling at the bedside, and transferring from the bed to the chair and ends with ambulation in the room. ${ }^{[30-32]}$

It was noted from the current study results, that the duration of mechanical ventilation was longer, the mortality rate was higher, the weaning difficulties was prominent, ICU stay was longer and the discharge rate from ICU was lower in patients with ICU-AW than others. Many studies agreed with these findings and found longer duration of mechanical ventilation among ICU-AW patients than others. ${ }^{[33-38]}$ Pooled analysis of 14 studies that enrolled patients after a specific period of mechanical ventilation suggests that the longer the exposure to mechanical ventilation the higher the incidence of ICUAW. ${ }^{[3]}$ Ali et al., ${ }^{[36]}$ Sharshar ${ }^{[13]}$ et al. and others ${ }^{[3,6,37,38]}$ reported ICU mortality of $31 \%-41 \%$ in ICU-AW patients, compared to $6 \%-10 \%$ no ICU-AW patients.

Results of the current study may be due to the spread of muscle weakness all over the body including the respiratory muscles. ICU-AW leads to autonomic dysfunction, which impaired patients' immunity that in turn caused infections; 
increased the durations of mechanical ventilation, increased the length of hospital stay and increased mortality rate. In general, autonomic dysfunction in critically ill patients may also increase mortality.

\section{Conclusion}

When critically ill patients admitted to ICU, nurses have the responsibility to maintain patients' safety and protect them from any harm of deteriorations/complications. The major risk factor for ICU-AW is immobility as patients are on strict bed rest in ICU. The other contributing factors for are patients' age, parenteral nutrition, and glucocorticoid $\&$ insulin therapy. ICU-AW preventive measures delivered by nurses were inadequate in ICU-AW group who depend on nurses to provide these measures, which resulted in occurrence of ICU-AW. Consequently, patients with ICU-AW may have many detrimental physiological consequences that lead to longer duration on mechanical ventilation, weaning difficulties, high length of ICU stay, high ICU mortality rate and low ICU discharge rate.

ICU-AW is one of many complications that ICU patients may develop because of ineffective mobilization, therefore, it is important to use evidences to reduce and/or eliminate the barriers of early mobilization. Mobilization of critically ill patients especially the hemodynamically unstable ones is a basic nursing care and it can be safe by using the evidence and protocols. ICU culture should be shifted from keeping patients on restrict bed rest to mobilizing patients as early as possible. Stakeholders' engagement is required for success is important to achieve mobilization safety protocols. Assessment of muscle weakness should be a part of the daily assessment of patients. In addition, longitudinal studies examining the natural history of ICU-AW in ICU survivors and the chronic critically ill are needed, as well as a deeper understanding of the associations between the ICU-AW and quality of life in these patients. Further researches are needed to examine ICU-AW in unconscious or sedated patients.

\section{Limitations of the study}

Data were collected in only 2 hospitals on convenient sample of 100 patients and this not enough sample to generalize the results. Subjects in the study were fully conscious and ICU-AW can occurred in unconscious or sedated patients therefore; more studies are needed to examine ICU-AW in these patients. In addition, there is a need to study extensively about the relation between nutrition and occurrence of ICU-AW.

\section{CONFlicts OF INTEREST Disclosure}

The authors declare that there is no competing interests.

\section{REFERENCES}

[1] Rukstele C. Making Strides in Preventing ICU-Acquired Weakness Involving Family in Early Progressive Mobility. Crit Care Nurs Q. 2013; 36(1): 141-7. PMid:23221450. http://dx.doi.org/10.10 97/CNQ.0b013e31827539cc

[2] Koo K. ICU-Acquired Weakness and Early Rehabilitation in the Critically Ill. JCOM. 2013; 20(5): 223-31.

[3] American thoracic society documents. An Official American Thoracic Society Clinical Practice Guideline: The Diagnosis of Intensive Care Unit-acquired Weakness in Adults. Am J Respir Crit Care Med. 2014; 190 (Iss 12): 1437-46.

[4] Hough C, Lieu B, Caldwell E. Manual muscle strength testing of critically ill patients: feasibility and interobserver agreement. Critical Care. 2011; 15: 43.

[5] Paternostro-Sluga T, Grim-Stieger M, Posch M, et al. Reliability and validity of the Medical Research Council (MRC) scale and a modified scale for testing muscle strength in patients with radial palsy. J Rehabil Med. 2008; 40(8): 665-71. PMid:19020701. http://dx.doi.org/10.2340/16501977-0235

[6] Kress J, Hall J. ICU-Acquired Weakness and Recovery from Critical Illness. N Engl J Med. 2014; 370(17): 1626-35. PMid:24758618. http://dx.doi.org/10.1056/NEJMra1209390

[7] Dammeyer J, Dickinson S, Packard D, et al. Building a Protocol to Guide Mobility in the ICU. Crit Care Nurs Q. 2013; 36(1): 37-49.
[8] Vollman K. Understanding Critically Ill Patients Hemodynamic Response to Mobilization Using the Evidence to Make It Safe and Feasible. Crit Care Nurs Q. 2013; 36(1): 17-27.

[9] Koo K. Survey of acute rehabilitation in Canadian intensive care units. Published MSc Thesis - McMaster University, School of Graduate Studies. 2012: 30-50.

[10] Sidiras G, Gerovasili V, Patsaki I, et al. Short and long term outcomes of ICU acquired weakness. Health science journal. 2013; 7(2): 188-200.

[11] Lee C, Fan E. ICU-acquired weakness: what is preventing its rehabilitation in critically ill patients? BMC Medicine. 2012; 10: 115.

[12] Herridge M, Tansey C, Matt A, et al. Canadian Critical Care Trials Group. Functional disability 5 years after acute respiratory distress syndrome. N Engl J Med. 2011; 364: 1293-304. PMid:21470008. http://dx.doi.org/10.1056/NEJMoa1011802

[13] Sharshar T, Bastuji-Garin S, Stevens R, et al. Presence and severity of intensive care unit-acquired paresis at time of awakening are associated with increased intensive care unit and hospital mortality. Crit Care Med. 2009; 37: 3047-53. PMid:19770751. http: //dx.doi.org/10.1097/CCM.0b013e3181b027e9

[14] Pearmain L, Herridge M. Outcomes after ARDS: a distinct group in the spectrum of disability after complex and protracted critical illness. Minerva Anestesiol. 2013; 79: 793-803.

[15] McWilliams D, Weblin J, Atkins G, et al. Enhancing rehabilitation of mechanically ventilated patients in the intensive care unit: A quality improvement project. Journal of Critical Care. 2015; 30: 13- 
8. PMid:25316527. http://dx.doi.org/10.1016/j.jcrc. 20 14.09 .018

[16] Timmerman R. A Mobility Protocol for Critically Ill Adults Dimensions of Critical Care Nursing. 2007; 26(5): 175-9.

[17] Burtin C, Clerckx B, Robbeets C, et al. Early exercise in critically ill patients enhances short-term functional recovery. Crit Care Med. 2009; 37(9): 2499-505. PMid:19623052. http://dx.doi .org/10. 1097/CCM. 0b013e3181a38937

[18] Nanas S, Kritikos K, Angelopoulos E, et al. Predisposing factors for critical illness polyneuromyopathy in a multidisciplinary intensive care unit. Acta Neurol Scand. 2008; 118(3): 175-81.

[19] Puthucheary Z, Rawal J, McPhail M, et al. Acute skeletal muscle wasting in critical illness. JAMA. 2013; 310: 1591-600. PMid:24108501. http://dx.doi.org/10.1001/jama.2013.2 78481

[20] Hermans G, Casaer M, Clerckx B, et al. Effect of tolerating macronutrient deficit on the development of intensive-care unit acquired weakness: a subanalysis of the EPaNIC trial. Lancet Respir Med. 2013; 1: 621-9.

[21] Needham DM, Dinglas VD, Bienvenu OJ, et al. One year outcomes in patients with acute lung injury randomised to initial trophic or full enteral feeding: prospective follow-up of EDEN randomised trial. BMJ. 2013; 346: f1532.

[22] Morris PE. Moving our critically ill patients: mobility barriers and benefits. Crit Care Clin. 2007; 23: 1-20. PMid:17307113. http://dx.doi.org/10.1016/j.ccc.2006.11.003

[23] Winkelman C, Higgins P, Chen Y. Activity in chronically critically ill. Dimens Crit Care Nurs. 2005; 24(6): 281-90. http: //dx.doi.org/10.1097/00003465-200511000-00011

[24] Patient turning and repositioning: current methods \& challenges, a WOCN perspective. Acailable from: www . sageproducts . com/. /WOCN\%20White\%20Paper_Aug2012.pdf

[25] Ahrens T, Kollef M, Stewart J, et al. Effects of kinetic therapy on pulmonary complications. Am J Crit Care. 2004; 13: 376-82.

[26] Goldhill D, Imhoff M, McLean B, et al. Rotational bed therapy to prevent and treat respiratory complications: a review and meta-analysis. Am J Crit Care. 2007; 16(1): 50-61.

[27] Swandneer-Culpepper L, Skaggs R, VanGilder C. The impact of continuous lateral rotation therapy in overall clinical and financial outcomes of critically ill patients. Crit Care Nurse Q. 2008; 31(3): 270-9.

[28] Freeman R, Maley K. Mobilization of Intensive Care Cardiac Surgery Patients on Mechanical Circulatory Support. Crit Care Nurs Q. 2013; 36(1): 73-88

[29] Bailey P, Thomsen G, Spuhler V, et al. Early activity is feasible and safe in respiratory failure patients. Crit Care Med. 2007; 35(1): $139-45$.

[30] Vollman K. Introduction to progressive mobility. Crit Care Nurse. 2010; 30(2): S3-S5.

[31] Morris P, Goad A, Thompson C, et al. Early intensive care unit mobility therapy in the treatment of acute respiratory failure. Crit Care Med. 2008; 36(8): 2238-43. PMid:18596631. http://dx.doi .org /10.1097/CCM.0b013e318180b90e

[32] Havey R, Herriman E. Guarding the gut: early mobility after abdominal surgery. CCNQ. 2013; 36(1): 63-72.

[33] Winkelman C, Peereboom K. Staff-perceived barriers and facilitators. Crit Care Nurse. 2010; 30: 13-16. PMid:20360441. http: $/ /$ dx.doi.org/10.4037/ccn2010393

[34] Bassett R, Vollman K, Brandwene L, et al. Integrating a multidisciplinary mobility program into intensive care practice (IMMPTP): a multicenter collaborative. Intensive Crit Care Nurs. 2012; 28(2): 88-97. PMid:22227355. http://dx.doi.org/10.1016/j.iccn. 2011.12 .001

[35] Vollman K. Hemodynamic instability: is it really a barrier to turning critically ill patients? Crit Care Nurse. 2012; 32(1): 70-5. PMid:22298720. http://dx.doi.org/10.4037/ccn2012765

[36] Ali N, O'Brien J, Hoffmann S, et al. Midwest Critical Care Consortium. Acquired weakness, handgrip strength, and mortality in critically ill patients. Am J Respir Crit Care Med. 2008; 178: 2618. PMid:18511703. http://dx.doi.org/10.1164/rccm. 2007 12-18290C

[37] Batt J, Santos C, Cameron J, et al. Intensive care unit acquired weakness: clinical phenotypes and molecular mechanisms. Am J Respir Crit Care Med. 2013; 187: 238-46. PMid:23204256. http: //dx.doi.org/10.1164/rccm.201205-0954SO

[38] Fan E, Dowdy D, Colantuoni E, et al. Physical complications in acute lung injury survivors: a twoyear longitudinal prospective study. Crit Care Med. 2014; 42: 849-59. PMid:24247473. http: //dx.doi.org/10.1097/CCM.0000000000000040 